\title{
Global Positive Periodic Solutions of Generalized $n$-Species Competition Systems with Multiple Delays and Impulses
}

\author{
Zhenguo Luo ${ }^{1,2}$ and Liping $\mathrm{Luo}^{2}$ \\ ${ }^{1}$ Department of Mathematics, National University of Defense Technology, Changsha 410073, China \\ ${ }^{2}$ Department of Mathematics, Hengyang Normal University, Hengyang, Hunan 421008, China
}

Correspondence should be addressed to Zhenguo Luo; robert186@163.com

Received 20 April 2013; Accepted 18 June 2013

Academic Editor: M. Victoria Otero-Espinar

Copyright (c) 2013 Z. Luo and L. Luo. This is an open access article distributed under the Creative Commons Attribution License, which permits unrestricted use, distribution, and reproduction in any medium, provided the original work is properly cited.

By applying the fixed point theorem in a cone of Banach space, we obtain an easily verifiable necessary and sufficient condition for the existence of positive periodic solutions of two kinds of generalized $n$-species competition systems with multiple delays and impulses as follows: $x_{i}^{\prime}(t)=x_{i}(t)\left[a_{i}(t)-b_{i}(t) x_{i}(t)-\sum_{j=1}^{n} c_{i j}(t) x_{i}\left(t-\tau_{i j}(t)\right)-\sum_{j=1}^{n} d_{i j}(t) x_{j}\left(t-\gamma_{i j}(t)\right)-\sum_{j=1}^{n} e_{i j}(t) \int_{-\sigma_{i j}}^{0} f_{i j}(s) x_{j}(t+\right.$ s)ds], a.e., $t>0, t \neq t_{k}, k \in Z_{+}, i=1,2, \ldots, n ; x_{i}\left(t_{k}^{+}\right)-x_{i}\left(t_{k}^{-}\right)=\theta_{i k} x_{i}\left(t_{k}\right), i=1,2, \ldots, n, k \in Z_{+}$; and $x_{i}^{\prime}(t)=x_{i}(t)\left[a_{i}(t)-\right.$ $\left.b_{i}(t) x_{i}(t)+\sum_{j=1}^{n} c_{i j}(t) x_{i}\left(t-\tau_{i j}(t)\right)-\sum_{j=1}^{n} d_{i j}(t) x_{j}\left(t-\gamma_{i j}(t)\right)-\sum_{j=1}^{n} e_{i j}(t) \int_{-\sigma_{i j}}^{0} f_{i j}(s) x_{j}(t+s) d s\right]$, a.e., $t>0, t \neq t_{k}, k \in Z_{+}, i=1,2, \ldots, n$; $x_{i}\left(t_{k}^{+}\right)-x_{i}\left(t_{k}^{-}\right)=\theta_{i k} x_{i}\left(t_{k}\right), i=1,2, \ldots, n, k \in Z_{+}$. It improves and generalizes a series of the well-known sufficiency theorems in the literature about the problems mentioned previously.

\section{Introduction}

Let $R=(-\infty,+\infty), R_{+}=[0,+\infty), R_{-}=(-\infty, 0], R^{n}=$ $\left\{\left(x_{1}, \ldots, x_{n}\right)^{T}: x_{i} \geq 0,1 \leq i \leq n\right\}, J \subset R, Z_{+}=\{1,2,3, \ldots\}$, respectively, and let $\omega>0$ be a constant and $C_{\omega}=\{x \mid x \in$ $C(R, R), x(t+\omega)=x(t)\}$, with the norm defined by $|x|_{0}=$ $\max _{t \in[0, \omega]}|x(t)| ; C_{\omega}^{1}=\left\{x \mid x \in C^{1}(R, R), x(t+\omega)=x(t)\right\}$, with the norm defined by $\|x\|=\max _{t \in[0, \omega]}\left\{|x|_{0},\left|x^{\prime}\right|_{0}\right\} ; P C=$ $\left\{x \mid x: R \rightarrow R^{+}, \lim _{s \rightarrow t} x(s)=x(t)\right.$, if $t \neq t_{k}, \lim _{t \rightarrow t_{k}^{-}} x(t)=$ $x\left(t_{k}\right), \lim _{t \rightarrow t_{k}^{+}} x(t)$ exists, $\left.k \in Z_{+}\right\} ; P C^{1}=\{x \mid x: R \rightarrow$ $\left.R^{+}, x^{\prime} \in P C\right\} ; P C_{\omega}=\{x \mid x \in P C, x(t+\omega)=x(t)\}$, with the norm defined by $|x|_{0}=\max _{t \in[0, \omega]}|x(t)| ; P C_{\omega}^{1}=$ $\left\{x \mid x \in P C^{1}, x(t+\omega)=x(t)\right\}$, with the norm defined by $\|x\|=\max _{t \in[0, \omega]}\left\{|x|_{0},\left|x^{\prime}\right|_{0}\right\} ; g^{M}=\max _{t \in[0, \omega]}\{g(t)\}, g^{L}=$ $\min _{t \in[0, \omega]}\{g(t)\}$, for any $g(t) \in P C_{\omega}$.

The theory of impulsive delay differential equations is emerging as an important area of investigation, since it is a lot richer than the corresponding theory of nonimpulsive delay differential equations. Many evolution processes in nature are characterized by the fact that at certain moments of time they experience abrupt change of state. That was the reason for the development of the theory of impulsive differential equations and impulsive delay differential equations, see the monographs [1-4]. In recent years, the existence theory of positive periodic solutions of delay differential equations with impulsive effects or without impulsive effects has been an object of active research, which is referred to as [5-12]. However, only a little work has been done on the existence of positive periodic solutions to the high-dimension impulsive differential equations based on the theory in cones. Motivated by this, in this paper, we mainly consider the following two classes of $n$-species Lotka-Volterra competitive systems with multiple delays and impulses:

$$
\begin{aligned}
x_{i}^{\prime}(t)=x_{i}(t) & {\left[a_{i}(t)-b_{i}(t) x_{i}(t)-\sum_{j=1}^{n} c_{i j}(t) x_{i}\left(t-\tau_{i j}(t)\right)\right.} \\
& -\sum_{j=1}^{n} d_{i j}(t) x_{j}\left(t-\gamma_{i j}(t)\right) \\
& \left.-\sum_{j=1}^{n} e_{i j}(t) \int_{-\sigma_{i j}}^{0} f_{i j}(s) x_{j}(t+s) d s\right],
\end{aligned}
$$




$$
\begin{gathered}
\text { a.e., } t>0, t \neq t_{k}, k \in Z_{+}, i=1,2, \ldots, n, \\
x_{i}\left(t_{k}^{+}\right)-x_{i}\left(t_{k}^{-}\right)=\theta_{i k} x_{i}\left(t_{k}\right), \quad i=1,2, \ldots, n ; k \in Z_{+} ; \\
x_{i}^{\prime}(t)=x_{i}(t)\left[\begin{array}{l}
a_{i}(t)-b_{i}(t) x_{i}(t)+\sum_{j=1}^{n} c_{i j}(t) x_{i}\left(t-\tau_{i j}(t)\right) \\
-\sum_{j=1}^{n} d_{i j}(t) x_{j}\left(t-\gamma_{i j}(t)\right) \\
\left.-\sum_{j=1}^{n} e_{i j}(t) \int_{-\sigma_{i j}}^{0} f_{i j}(s) x_{j}(t+s) d s\right], \\
\text { a.e., } t>0, t \neq t_{k}, k \in Z_{+}, i=1,2, \ldots, n, \\
x_{i}\left(t_{k}^{+}\right)-x_{i}\left(t_{k}^{-}\right)=\theta_{i k} x_{i}\left(t_{k}\right), \quad i=1,2, \ldots, n ; k \in Z_{+},
\end{array}\right.
\end{gathered}
$$

where $a_{i}(t), b_{i}(t), c_{i j}(t), d_{i j}(t), e_{i j}(t), \tau_{i j}(t), \gamma_{i j}(t) \in P C_{\omega}, i, j=$ $1,2, \ldots, n$, with $\overline{a_{i}}=(1 / \omega) \int_{0}^{\omega} a_{i}(t) d t>0$. Moreover, $f_{i j} \in$ $P C, \sigma_{i j}>0$ is a constant, and $\int_{-\sigma_{i j}}^{0} f_{i j}(s) d s=1$. Furthermore, $\Delta x_{i}\left(t_{k}\right)=x_{i}\left(t_{k}^{+}\right)-x_{i}\left(t_{k}\right), i=1,2, \ldots, n$ (here $x\left(t_{k}^{+}\right)$represents the right limit of $x(t)$ at the point $\left.t_{k}\right) ; \theta_{k}=\left(\theta_{1 k}, \theta_{2 k}, \ldots, \theta_{n k}\right) \in$ $C\left(R_{+}^{n}, R_{-}^{n}\right)$; that is, $x_{i}(t)$ changes decreasingly suddenly at times $t_{k}$. We assume that there exists an integer $p>0$ such that $t_{k+p}=t_{k}+\omega, \theta_{i(k+p)}=\theta_{i k}, i=1,2, \ldots, n$, where $0<t_{1}<t_{2}<\cdots<t_{p}<\omega$.

References [13, 14], G. Seifert investigated the following periodic single-species population growth models with periodic delay:

$$
y^{\prime}(t)=y(t)[r(t)-a(t) y(t)-b(t) y(t-\tau(t))] .
$$

They had assumed that the net birth $r(t)$, the self-inhibition rate $a(t)$, and the delay $\tau(t)$ are continuously differentiable $\omega$ periodic functions, and $r(t)>0, a(t)>0, b(t) \geq 0, \tau(t) \geq 0$, for $t \in R$. The negative feedback term $-b(t) y(t-\tau(t))$ in the average growth rate of species has a negative time delay (the sign of the time delay term is negative), which can be regarded as the deleterious effect of time delay on a species growth rate. They had derived sufficient conditions for the existence and global attractivity of positive periodic solutions of system (3). But the discussion of global attractivity is only confined to the special case when the periodic delay is constant.

In [15], Freedman and $\mathrm{Wu}$ proposed the following periodic single-species population growth models with periodic delay:

$$
y^{\prime}(t)=y(t)[r(t)-a(t) y(t)+b(t) y(t-\tau(t))] .
$$

They had assumed that the net birth $r(t)$, the self-inhibition rate $a(t)$, and the delay $\tau(t)$ are continuously differentiable $\omega$ periodic functions, and $r(t)>0, a(t)>0, b(t) \geq 0, \tau(t) \geq 0$ for $t \in R$. The positive feedback term $b(t) y(t-\tau(t))$ in the average growth rate of species has a positive time delay (the sign of the time delay term is positive), which is a delay due to gestation. They had established sufficient conditions which guarantee that system (4) has a positive periodic solution which is globally asymptotically stable.

References $[16,17]$ have studied the following two-species competitive system without delay:

$$
\begin{aligned}
& y_{1}^{\prime}(t)=y_{1}(t)\left[r_{1}(t)-a_{1}(t) y_{1}(t)-c_{1}(t) y_{2}(t)\right] \\
& y_{2}^{\prime}(t)=y_{2}(t)\left[r_{2}(t)-a_{2}(t) y_{2}(t)-c_{2}(t) y_{1}(t)\right] .
\end{aligned}
$$

They had derived sufficient conditions for the existence and global attractivity of positive periodic solutions of system (5) by using differential inequalities and topological degree, respectively. In fact, in many practical situations the time delay occurs so often. A more realistic model should include some of the past states of the system. Motivated by the previous ideas, Liu et al. [18] considered two corresponding periodic Lotka-Volterra competitive systems involving multiple delays:

$$
\begin{aligned}
& y_{1}^{\prime}(t)=y_{1}(t)\left[r_{1}(t)-a_{1}(t) y_{1}(t)+\sum_{i=1}^{n} b_{1 i}(t) y_{1}\left(t-\tau_{i}(t)\right)\right. \\
& \left.-\sum_{j=1}^{m} c_{1 j}(t) y_{2}\left(t-\rho_{j}(t)\right)\right] \\
& y_{2}^{\prime}(t)=y_{2}(t)\left[r_{2}(t)-a_{2}(t) y_{2}(t)+\sum_{j=1}^{m} b_{2 j}(t) y_{2}\left(t-\eta_{j}(t)\right)\right. \\
& \left.-\sum_{i=1}^{n} c_{2 i}(t) y_{1}\left(t-\sigma_{i}(t)\right)\right] \\
& y_{1}^{\prime}(t)=y_{1}(t)\left[r_{1}(t)-a_{1}(t) y_{1}(t)-\sum_{i=1}^{n} b_{1 i}(t) y_{1}\left(t-\tau_{i}(t)\right)\right. \\
& \left.-\sum_{j=1}^{m} c_{1 j}(t) y_{2}\left(t-\rho_{j}(t)\right)\right] \\
& y_{2}^{\prime}(t)=y_{2}(t)\left[r_{2}(t)-a_{2}(t) y_{2}(t)-\sum_{j=1}^{m} b_{2 j}(t) y_{2}\left(t-\eta_{j}(t)\right)\right. \\
& \left.-\sum_{i=1}^{n} c_{2 i}(t) y_{1}\left(t-\sigma_{i}(t)\right)\right] \text {, }
\end{aligned}
$$

where $b_{1 i}(t), b_{2 j}(t) \in C(R,[0,+\infty)), a_{1}(t), a_{2}(t), c_{1 j}(t), c_{2 i}(t) \in$ $C(R,[0,+\infty)), \tau_{i}(t), \rho_{j}(t), \eta_{j}(t), \sigma_{i}(t) \in C^{1}(R,[0,+\infty))(i=$ $1,2, \ldots, n ; j=1,2, \ldots, m)$ are $\omega$-periodic functions. Here, the intrinsic growth rates $r_{k}(t) \in C(R, R)$ are $\omega$-periodic functions with $\int_{0}^{\omega} r_{k}(t) d t>0, k=1,2$. They had derived the same criteria for the existence and globally asymptotic stability of positive periodic solutions of the previous two competitive systems by using Gaines and Mawhin's continuation theorem of coincidence degree theory and by means of a suitable Lyapunov functional. However, to this day, no scholars had done 
works on the existence of positive periodic solution of (1) and (2). One could easily see that systems (3)-(6) are all special cases of system (1) and (2). tions:

Throughout the paper, we make the following assump-

$\left(\mathrm{H}_{1}\right) a_{i}(t), b_{i}(t), c_{i j}(t), d_{i j}(t), e_{i j}(t), \tau_{i j}(t), \gamma_{i j}(t) \in P C_{\omega}, i, j=$ $1,2, \ldots, n$, with $\overline{a_{i}}=(1 / \omega) \int_{0}^{\omega} a_{i}(t) d t>0, \tau=$ $\max _{1 \leq i \leq n} \max _{1 \leq j \leq n}\left\{\tau_{i j}(t), \gamma_{i j}(t)\right\}$;

$\left(\mathrm{H}_{2}\right)\left\{t_{k}\right\}, k \in Z_{+}$satisfies $0<t_{1}<t_{2}<\cdots<t_{k}<\cdots$ and $\lim _{k \rightarrow+\infty} t_{k}=+\infty$;

$\left(\mathrm{H}_{3}\right)\left\{\theta_{i k}\right\}$ is a real sequence with $\Delta_{i k}=\theta_{i k}+1>0, i=$ $1,2, \ldots, n, k \in Z_{+}$;

$\left(\mathrm{H}_{4}\right) \prod_{0<t_{k}<t} \Delta_{i k}$ is a $\omega$-periodic function.

To conclude this section, we summarize in the following a few definitions and lemmas that will be needed in our arguments.

Definition 1 (see [1]). A function $x_{i}: R \rightarrow(0,+\infty)$ is said to be a positive solution of (1) and (2), if the following conditions are satisfied:

(a) $x_{i}(t)$ is absolutely continuous on each $\left(t_{k}, t_{k+1}\right)$;

(b) for each $k \in Z_{+}, x_{i}\left(t_{k}^{+}\right)$and $x_{i}\left(t_{k}^{-}\right)$exist and $x_{i}\left(t_{k}^{-}\right)=$ $x_{i}\left(t_{k}\right)$;

(c) $x_{i}(t)$ satisfies the first equation of (1) and (2) for almost everywhere (for short a.e.) in $[0, \infty] \backslash\left\{t_{k}\right\}$ and satisfies $x_{i}\left(t_{k}^{+}\right)=\Delta_{i k} x_{i}\left(t_{k}\right)$ for $t=t_{k}, k \in Z_{+}=$ $\{1,2, \ldots\}$.

Under the previous hypotheses $\left(\mathrm{H}_{1}\right)-\left(\mathrm{H}_{4}\right)$, we consider the following two classes of nonimpulsive Lotka-Volterra competitive systems:

$$
\begin{aligned}
& \frac{d y_{i}(t)}{d t} \\
& =y_{i}(t)\left[a_{i}(t)-B_{i}(t) y_{i}(t)-\sum_{j=1}^{n} C_{i j}(t) y_{i}\left(t-\tau_{i j}(t)\right)\right. \\
& \\
& \quad-\sum_{j=1}^{n} D_{i j}(t) y_{j}\left(t-\gamma_{i j}(t)\right)-\sum_{j=1}^{n} E_{i j}(t) \\
& \\
& \left.\quad \times \int_{-\sigma_{i j}}^{0} f_{i j}(s) y_{j}(t+s) d s\right], \quad \text { a.e., } t \geq 0,
\end{aligned}
$$

$$
\begin{aligned}
& \frac{d y_{i}(t)}{d t} \\
& =y_{i}(t)\left[a_{i}(t)-B_{i}(t) y_{i}(t)+\sum_{j=1}^{n} C_{i j}(t) y_{i}\left(t-\tau_{i j}(t)\right)\right. \\
& \quad-\sum_{j=1}^{n} D_{i j}(t) y_{j}\left(t-\gamma_{i j}(t)\right)-\sum_{j=1}^{n} E_{i j}(t) \\
& \left.\quad \times \int_{-\sigma_{i j}}^{0} f_{i j}(s) y_{j}(t+s) d s\right], \quad \text { a.e., } t \geq 0,
\end{aligned}
$$

where

$$
\begin{gathered}
B_{i}(t)=b_{i}(t) \prod_{0<t_{k}<t} \Delta_{i k}, \\
C_{i j}(t)=c_{i j}(t) \prod_{0<t_{k}<t-\tau_{i j}(t)} \Delta_{i k}, \\
D_{i j}(t)=d_{i j}(t) \prod_{0<t_{k}<t-\gamma_{i j}(t)} \Delta_{i k}, \\
E_{i j}(t)=e_{i j}(t) \prod_{0<t_{k}<t-\sigma_{i j}} \Delta_{i k} .
\end{gathered}
$$

By a solution $y(t)=\left(y_{1}(t), \ldots, y_{n}(t)\right)^{T}$ of $(7)$ and (8), it means an absolutely continuous function $y(t)=\left(y_{1}(t), \ldots, y_{n}(t)\right)^{T}$ defined on $[-\tau, 0]$ that satisfies (7) and (8).

The following lemmas will be used in the proofs of our results. The proof of the first lemma is similar to that of Theorem 1 in [5].

Lemma 2. Suppose that $\left(H_{1}\right)-\left(H_{4}\right)$ hold. Then

(1) if $y_{i}(t)(i=1,2, \ldots, n)$ are solutions of (7) and (8) on $[-\tau,+\infty)$, then $x_{i}(t)=\prod_{0<t_{k}<t} \Delta_{i k} y_{i}(t)(i=$ $1,2, \ldots, n)$ are solutions of $(1)$ and $(2)$ on $[-\tau,+\infty)$;

(2) if $x_{i}(t)(i=1,2, \ldots, n)$ are solutions of (1) and (2) on $[-\tau,+\infty)$, then $y_{i}(t)=\prod_{0<t_{k}<t} \Delta_{i k}^{-1} x_{i}(t)(i=$ $1,2, \ldots, n)$ are solutions of $(7)$ and $(8)$ on $[-\tau,+\infty)$.

Proof. (1) It is easy to see that $x_{i}(t)=\prod_{0<t_{k}<t} \Delta_{i k} y_{i}(t)(i=$ $1,2, \ldots, n)$ are absolutely continuous on every interval $\left(t_{k}, t_{k+1}\right], t \neq t_{k}, k=1,2, \ldots$,

$$
\begin{aligned}
x_{i}^{\prime}(t)-x_{i}(t)[ & a_{i}(t)-b_{i}(t) x_{i}(t)-\sum_{j=1}^{n} c_{i j}(t) x_{i}\left(t-\tau_{i j}(t)\right) \\
& -\sum_{j=1}^{n} d_{i j}(t) x_{j}\left(t-\gamma_{i j}(t)\right)-\sum_{j=1}^{n} e_{i j}(t) \\
& \left.\times \int_{-\sigma_{i j}}^{0} f_{i j}(s) x_{j}(t+s) d s\right]
\end{aligned}
$$




$$
\begin{aligned}
& =\prod_{0<t_{k}<t} \Delta_{i k} y_{i}^{\prime}(t)-\prod_{0<t_{k}<t} \Delta_{i k} y_{i}(t) \\
& \times\left[a_{i}(t)-b_{i}(t) \prod_{0<t_{k}<t} \Delta_{i k} y_{i}(t)-\sum_{j=1}^{n} c_{i j}(t)\right. \\
& \times \prod_{0<t_{k}<t-\tau_{i j}(t)} \Delta_{i k} y_{i}\left(t-\tau_{i j}(t)\right)-\sum_{j=1}^{n} d_{i j}(t) \\
& \times \prod_{0<t_{k}<t-\gamma_{i j}(t)} \Delta_{i k} y_{j}\left(t-\gamma_{i j}(t)\right) \\
& \left.-\sum_{j=1}^{n} \prod_{0<t_{k}<t-\sigma_{i j}} \Delta_{i k} e_{i j}(t) \int_{-\sigma_{i j}}^{0} f_{i j}(s) y_{j}(t+s) d s\right] \\
& =\prod_{0<t_{k}<t} \Delta_{i k}\left\{y_{i}^{\prime}(t)-y_{i}(t)\right. \\
& \times\left[a_{i}(t)-b_{i}(t) \prod_{0<t_{k}<t} \Delta_{i k} y_{i}(t)\right. \\
& -\sum_{j=1}^{n} c_{i j}(t) \prod_{0<t_{k}<t-\tau_{i j}(t)} \Delta_{i k} y_{i} \\
& \times\left(t-\tau_{i j}(t)\right)-\sum_{j=1}^{n} d_{i j}(t) \\
& \times \prod_{0<t_{k}<t-\gamma_{i j}(t)} \Delta_{i k} y_{j}\left(t-\gamma_{i j}(t)\right) \\
& -\sum_{j=1}^{n} \prod_{0<t_{k}<t-\sigma_{i j}} \Delta_{i k} e_{i j}(t) \int_{-\sigma_{i j}}^{0} f_{i j}(s) y_{j} \\
& \times(t+s) d s]\} \\
& =\prod_{0<t_{k}<t} \Delta_{i k}\left\{y_{i}^{\prime}(t)-y_{i}(t)\right. \\
& \times\left[a_{i}(t)-B_{i}(t) y_{i}(t)-\sum_{j=1}^{n} C_{i j}(t) y_{i}\right. \\
& \times\left(t-\tau_{i j}(t)\right)-\sum_{j=1}^{n} D_{i j}(t) y_{j}\left(t-\gamma_{i j}(t)\right) \\
& \left.\left.-\sum_{j=1}^{n} E_{i j}(t) \int_{-\sigma_{i j}}^{0} f_{i j}(s) y_{j}(t+s) d s\right]\right\}
\end{aligned}
$$

$=0$.

On the other hand, for any $t=t_{k}, k=1,2, \ldots$,

$$
\begin{gathered}
x_{i}\left(t_{k}^{+}\right)=\lim _{t \rightarrow t_{k 0<t_{j}<t}} \prod_{i k} y_{i}(t)=\prod_{0<t_{j} \leq t_{k}} \Delta_{i k} y_{i}\left(t_{k}\right), \\
x_{i}\left(t_{k}\right)=\prod_{0<t_{j}<t_{k}} \Delta_{i k} y_{i}\left(t_{k}\right), \quad i=1,2, \ldots, n ;
\end{gathered}
$$

thus

$$
x_{i}\left(t_{k}^{+}\right)=\Delta_{i k} x_{i}\left(t_{k}\right), \quad i=1,2, \ldots, n, k=1,2, \ldots
$$

It follows from $(10)-(12)$ that $x_{i}(t)(i=1,2, \ldots, n)$ are solutions of (1). Similarly, if $y_{i}(t)(i=1,2, \ldots, n)$ are solutions of (8), we can prove that $x_{i}(t)(i=1,2, \ldots, n)$ are solutions of (2).

(2) Since $x_{i}(t)=\prod_{0<t_{k}<t} \Delta_{i k} y_{i}(t)$ is absolutely continuous on every interval $\left(t_{k}, t_{k+1}\right], t \neq t_{k}, k=1,2, \ldots$, and in view of (12), it follows that for any $k=1,2, \ldots$,

$$
\begin{aligned}
& y_{i}\left(t_{k}^{+}\right)=\prod_{0<t_{j} \leq t_{k}} \Delta_{i k}^{-1} x_{i}\left(t_{k}^{+}\right)=\prod_{0<t_{j}<t_{k}} \Delta_{i k}^{-1} x_{i}\left(t_{k}\right)=y_{i}\left(t_{k}\right), \\
& y_{i}\left(t_{k}^{-}\right)=\prod_{0<t_{j}<t_{k}} \Delta_{i k}^{-1} x_{i}\left(t_{k}^{-}\right)=\prod_{0<t_{j} \leq t_{k}^{-}} \Delta_{i k}^{-1} x_{i}\left(t_{k}^{-}\right)=y_{i}\left(t_{k}\right),
\end{aligned}
$$

which implies that $y_{i}(t)$ is continuous on $[-\tau,+\infty)$. It is easy to prove that $y_{i}(t)$ is absolutely continuous on $[-\tau,+\infty)$. Similar to the proof of (1), we can check that $y_{i}(t)=$ $\prod_{0<t_{k}<t} \Delta_{i k}^{-1} x_{i}(t)(i=1,2, \ldots, n)$ are solutions of $(7)$ on $[-\tau,+\infty)$. Similarly, if $x_{i}(t), i=1,2, \ldots, n$, is a solution of $(2)$, we can prove that $y_{i}(t)(i=1,2, \ldots, n)$ are solutions of $(8)$. The proof of Lemma 2 is completed.

In the following section, we only discuss the existence of a periodic solution for (7) and (8).

Definition 3 (see [19]). Let $X$ be a real Banach space and $E$ a closed, nonempty subset of $X$. $E$ is said to be a cone if

(1) $\alpha x+\beta y \in E$ for all $x, y \in E$, and $\alpha, \beta>0$,

(2) $x,-x \in E$ imply $x=0$.

Lemma 4 (see Krasnoselskii [20], Deimling [21], and Guo and Lakshmikantham [22]). Let $E$ be a cone in a real Banach space $X$. Assume that $\Omega_{r_{1}}$ and $\Omega_{r_{2}}$ are open subsets of $X$ with $0 \in$ $\Omega_{r_{1}} \subset \overline{\Omega_{r_{1}}} \subset \Omega_{r_{2}}$, where $\Omega_{r_{i}}=\left\{x \in X:\|x\|<r_{i}\right\}, i=1$, 2. Let $T: E \cap\left(\Omega_{r_{2}} \backslash \overline{\Omega_{r_{1}}}\right) \rightarrow E$ be a continuous and completely continuous operator satisfying

(1) $\|T x\| \leq\|x\|$, for any $x \in E \cap \partial \Omega_{r_{1}}$;

(2) there exists $\phi \in E \backslash\{0\}$ such that $y \neq T y+\lambda y$, for any $y \in E \cap \partial \Omega_{r_{2}}$ and $\lambda>0$.

Then $T$ has fixed points in $E \cap\left(\overline{\Omega_{r_{2}}} \backslash \Omega_{r_{1}}\right)$. The same conclusion remains valid if (1) holds for any $x \in E \cap \partial \Omega_{r_{2}}$ and (2) holds for any $y \in E \cap \partial \Omega_{r_{1}}$ and $\lambda>0$.

The paper is organized as follows. In Section 2, firstly, we give some definitions and lemmas. Secondly, we derive a necessary and sufficient condition for at least one positive periodic solution of (1) which is established by using the fixedpoint theorem in the cone of Banach space under some conditions. In the following section, we also get a necessary and sufficient condition for at least one positive periodic solution of (2) that is also established by applying the fixed-point theorem in the cone of Banach space under some conditions. Finally, as applications, we study some particular cases of system (1) and (2) which have been investigated extensively in the references mentioned earlier. 


\section{Existence of Periodic Solution of (1)}

We establish the existence of positive periodic solutions of (1) by applying Lemma 4 . We will first make some preparations and list later a few preliminary results. Let $X=\{y=$ $\left.\left(y_{1}(t), y_{2}(t), \ldots, y_{n}(t)\right)^{T} \in C\left(R, R^{n}\right) \mid y(t+\omega)=y(t)\right\}$ with the norm $\|y\|=\sum_{i=1}^{n}\left|y_{i}\right|_{0},\left|y_{i}\right|_{0}=\sup _{t \in[0, \omega]}\left|y_{i}(t)\right|$. It is easy to verify that $(X,\|\cdot\|)$ is a Banach space.

We define an operator $\psi: X \rightarrow X$ as follows:

$$
(\psi y)(t)=\left((\psi y)_{1}(t),(\psi y)_{2}(t), \ldots,(\psi y)_{n}(t)\right)^{T},
$$

where

$$
\begin{aligned}
& (\psi y)_{i}(t) \\
& =\int_{t}^{t+\omega}\left\{G_{i}(t, \xi) y_{i}(\xi)\right. \\
& \times\left[B_{i}(\xi) y_{i}(\xi)+\sum_{j=1}^{n} C_{i j}(\xi) y_{i}\left(\xi-\tau_{i j}(\xi)\right)\right. \\
& \quad+\sum_{j=1}^{n} D_{i j}(\xi) y_{j}\left(\xi-\gamma_{i j}(\xi)\right) \\
& \left.\left.\quad+\sum_{j=1}^{n} E_{i j}(\xi) \int_{-\sigma_{i j}}^{0} f_{i j}(s) y_{j}(\xi+s) d s\right]\right\} d \xi, \\
& \quad i=1,2, \ldots, n,
\end{aligned}
$$

where

$$
\begin{array}{r}
G_{i}(t, \xi)=\frac{e^{-\int_{t}^{\xi} a_{i}(u)} d u}{1-e^{-\int_{0}^{\omega} a_{i}(u)} d u}=\frac{e^{\int_{\xi}^{t+\omega} a_{i}(u)} d u}{e^{\int_{0}^{\omega} a_{i}(u)} d u-1}, \\
G(t, \xi)=\operatorname{diag}\left[G_{1}(t, \xi), G_{2}(t, \xi), \ldots, G_{n}(t, \xi)\right], \\
t \leq \xi \leq t+\omega .
\end{array}
$$

It is clear that $G_{i}(t+\omega, \xi+\omega)=G_{i}(t, \xi), \partial G_{i}(t, \xi) / \partial t=$ $a_{i}(t) G_{i}(t, \xi), G_{i}(t, t)-G_{i}(t, t+\omega)=1$. In view of $\left(\mathrm{H}_{1}\right)$, we also define for $1 \leq i \leq n$

$$
\begin{gathered}
\alpha_{i}:=\min _{0 \leq t \leq \xi \leq \omega}\left|G_{i}(t, \xi)\right|=\frac{1}{e^{\int_{0}^{\omega} a_{i}(u) d u}-1}, \\
\beta_{i}:=\max _{0 \leq t \leq \xi \leq \omega}\left|G_{i}(t, \xi)\right|=\frac{e^{\int_{0}^{\omega} a_{i}(u) d u}}{e^{\int_{0}^{\omega} a_{i}(u) d u}-1}, \\
\alpha=\min _{1 \leq i \leq n} \alpha_{i}, \quad \beta=\max _{1 \leq i \leq n} \beta_{i}, \quad \sigma=\frac{\alpha}{\beta} \in(0,1) .
\end{gathered}
$$

Define $E$ to be a cone in $X$ by

$$
\begin{aligned}
E=\{y & =\left(y_{1}(t), y_{2}(t), \ldots, y_{n}(t)\right)^{T} \in X: y_{i}(t) \\
& \left.\geq \sigma\left|y_{i}\right|_{0}, t \in[0, \omega]\right\} .
\end{aligned}
$$

We easily verify that $E$ is a cone in $X$. For convenience of expressions, we define an operator $\varphi: E \rightarrow X$ by

$$
\begin{aligned}
(\varphi y)_{i}(t)=y_{i}(t)[ & B_{i}(t) y_{i}(t) \\
& +\sum_{j=1}^{n} C_{i j}(t) y_{i}\left(\xi-\tau_{i j}(t)\right) \\
& +\sum_{j=1}^{n} D_{i j}(t) y_{j}\left(t-\gamma_{i j}(t)\right) \\
& \left.+\sum_{j=1}^{n} E_{i j}(t) \int_{-\sigma_{i j}}^{0} f_{i j}(s) y_{j}(t+s) d s\right], \\
& i=1,2, \ldots, n .
\end{aligned}
$$

The proof of the main result in this paper is based on an application of Krasnoselskii fixed-point theorem in cones. To make use of fixed point theorem in the cone, firstly, we need to introduce some definitions and lemmas.

Lemma 5. Assume that $\left(H_{1}\right)-\left(H_{4}\right)$ hold. Then the solutions of (1) are defined on $[-\tau, \infty)$ and are positive.

Proof. By Lemma 2, we only need to prove that the solutions $y_{i}(t)(i=1,2, \ldots, n)$ of $(7)$ are defined on $[-\tau, \infty)$ and are positive on $[0, \infty)$. From (7), we have that for any $u_{i} \in$ $C\left([-\tau, 0), R^{+}\right), i=1,2,3, \ldots, n$, and $t>0$,

$$
\begin{aligned}
& y_{i}(t)=u_{i}(0) \exp \left\{\int _ { 0 } ^ { t } \left[a_{i}(\xi)-B_{i}(\xi) y_{i}(\xi)\right.\right.-\sum_{j=1}^{n} C_{i j}(\xi) y_{i}\left(\xi-\tau_{i j}(\xi)\right) \\
&-\sum_{j=1}^{n} D_{i j}(\xi) y_{j}\left(\xi-\gamma_{i j}(\xi)\right) \\
&-\sum_{j=1}^{n} E_{i j}(\xi) \int_{-\sigma_{i j}}^{0} f_{i j}(s) y_{j} \\
&\times(\xi+s) d s] d \xi\}, \\
& i=1,2, \ldots, n .
\end{aligned}
$$

Therefore, $y_{i}(t)(i=1,2, \ldots, n)$ are defined on $[-\tau, \infty)$ and are positive on $[0, \infty)$. The proof of Lemma 5 is complete.

Lemma 6. Assume that $\left(H_{1}\right)-\left(H_{4}\right)$ hold. Then $\psi: E \rightarrow E$ is well defined. 
Proof. In view of the definitions of $E$ and $F$, for any $y \in E$, we have

$$
\begin{aligned}
(\psi y)_{i}(t) & =\int_{t}^{t+\omega} G_{i}(t, \xi)(\varphi y)_{i}(\xi) d \xi \\
(\psi y)_{i}(t+\omega) & =\int_{t+\omega}^{t+2 \omega} G_{i}(t, \xi+\omega)(\varphi y)_{i}(\xi+\omega) d \xi \\
& =\int_{t}^{t+\omega} G_{i}(t, \xi)(\varphi y)_{i}(\xi) d \xi \\
& =(\psi y)_{i}(t) .
\end{aligned}
$$

Therefore, $(\psi y) \in X$. Furthermore, for any $y \in E$, it follows from (15) that

$$
\left|(\psi y)_{i}\right|_{0} \leq \beta_{i} \int_{0}^{\omega}(\varphi y)_{i}(\xi) d \xi
$$

On the other hand, we obtain

$$
\begin{aligned}
(\psi y)_{i}(t) & \geq \alpha_{i} \int_{0}^{\omega}(\varphi y)_{i}(\xi) d \xi \\
& \geq \frac{\alpha_{i}}{\beta_{i}}\left|(\psi y)_{i}\right|_{0} \\
& \geq \sigma\left|(\psi y)_{i}\right|_{0} .
\end{aligned}
$$

Therefore, $\psi y \in E$. The proof of Lemma 6 is complete.

Lemma 7. The operator $\psi: E \rightarrow E$ is continuous and completely continuous.

Proof. By using a standard argument one can show that $\psi$ is continuous on $E$. Now, we show that $\psi$ is completely continuous. Let $r$ be any positive constant and $S_{r}=\left\{y \in X:\left|y_{i}\right|_{0} \leq r\right\}$ a bounded set. For any $y \in S_{r}$, by (15) we have

$$
\begin{aligned}
& \left|(\psi y)_{i}\right|_{0} \\
& \leq \beta_{i} \int_{0}^{\omega}(\varphi y)_{i}(\xi) d \xi \\
& =\beta_{i} \int_{0}^{\omega} y_{i}(t)\left[B_{i}(t) y_{i}(t)+\sum_{j=1}^{n} C_{i j}(t) y_{i}\left(\xi-\tau_{i j}(t)\right)\right. \\
& +\sum_{j=1}^{n} D_{i j}(t) y_{j}\left(t-\gamma_{i j}(t)\right) \\
& \left.+\sum_{j=1}^{n} e_{i j}(t) \int_{-\sigma_{i j}}^{0} F_{i j}(s) y_{j}(t+s) d s\right] d \xi \\
& \leq \omega \beta_{i} r^{2}\left[B_{i}^{M}+\sum_{j=1}^{n}\left(C_{i j}^{M}+D_{i j}^{M}+E_{i j}^{M}\right)\right]:=R_{i} .
\end{aligned}
$$

Therefore, for any $y \in S_{r}$, we obtain

$$
\|\psi y\|=\sum_{i=1}^{n}\left|(\psi y)_{i}\right|_{0} \leq \sum_{i=1}^{n} R_{i}:=R,
$$

which implies that $\psi\left(S_{r}\right)$ is a uniformly bounded set. On the other hand, in view of the definitions of $\psi$ and $\varphi$, we have

$$
\begin{aligned}
\frac{d\left[(\psi y)_{i}(t)\right]}{d t}= & G_{i}(t, t+\omega)(\varphi y)_{i}(t+\omega)-G_{i}(t, t)(\varphi y)_{i}(t) \\
& +\int_{t}^{t+\omega} \frac{d G_{i}(t, \xi)}{d t}(\varphi y)_{i}(\xi) d \xi \\
& =-(\varphi y)_{i}(t)+a_{i}(t) \int_{t}^{t+\omega} G_{i}(t, \xi)(\varphi y)_{i}(\xi) d \xi \\
& =a_{i}(t)(\psi y)_{i}(t)-(\varphi y)_{i}(t) .
\end{aligned}
$$

Again, from (15), we obtain

$$
\begin{aligned}
\left|\frac{d\left[(\psi y)_{i}(t)\right]}{d t}\right| \leq a_{i}^{M} R_{i}+r^{2}\left[B_{i}^{M}+\sum_{j=1}^{n}\left(C_{i j}^{M}+D_{i j}^{M}+E_{i j}^{M}\right)\right] \\
:=\overline{R_{i}} \leq M:=\max _{i \in[1, n]}\left\{\overline{R_{i}}\right\},
\end{aligned}
$$

which implies that $d\left[(\psi y)_{i}(t)\right] / d t$, for any $y \in S_{r}$, is also uniformly bounded. Hence, $\psi\left(S_{r}\right) \subset X$ is a family of uniformly bounded and equicontinuous functions. By the wellknown Ascoli-Arzela theorem, we know that the operator $\psi$ is completely continuous. The proof of Lemma 7 is complete.

Lemma 8. Assume that $\left(H_{1}\right)-\left(H_{4}\right)$ hold. The existence of positive $\omega$-periodic solution of (7) is equivalent to that of nonzero fixed point of $\psi$ in $E$.

Proof. Assume that $y=\left(y_{1}(t), y_{2}(t), \ldots, y_{n}(t)\right)^{T} \in X$ is a periodic solution of (7). Then, we have

$$
\begin{aligned}
& {\left[y_{i}(t) e^{-\int_{0}^{t} a_{i}(u) d u}\right]^{\prime} } \\
&=-e^{-\int_{0}^{t} a_{i}(u) d u} y_{i}(t)\left[B_{i}(t) y_{i}(t)+\sum_{j=1}^{n} C_{i j}(t) y_{i}\left(t-\tau_{i j}(t)\right)\right. \\
&+\sum_{j=1}^{n} D_{i j}(t) y_{j}\left(t-\gamma_{i j}(t)\right) \\
&\left.+\sum_{j=1}^{n} E_{i j}(t) \int_{-\sigma_{i j}}^{0} f_{i j}(s) y_{j}(t+s) d s\right],
\end{aligned}
$$


Integrating the previous equation over $[t, t+\omega]$, we can have

$$
\begin{aligned}
& {\left.\left[y_{i}(\xi) e^{-\int_{0}^{\xi} a_{i}(u) d u}\right]\right|_{t} ^{t+\omega}} \\
& =-\int_{t}^{t+\omega}\left\{e^{-\int_{0}^{\xi} a_{i}(u) d u} y_{i}(\xi)\right. \\
& \times\left[B_{i}(\xi) y_{i}(\xi)+\sum_{j=1}^{n} C_{i j}(\xi) y_{i}\left(\xi-\tau_{i j}(\xi)\right)\right. \\
& +\sum_{j=1}^{n} D_{i j}(\xi) y_{j}\left(\xi-\gamma_{i j}(\xi)\right) \\
& \left.\left.+\sum_{j=1}^{n} E_{i j}(\xi) \int_{-\sigma_{i j}}^{0} f_{i j}(s) y_{j}(\xi+s) d s\right]\right\} d \xi, \\
& i=1,2, \ldots, n \text {. }
\end{aligned}
$$

Therefore, we have

$$
\begin{aligned}
y_{i}(t) e^{-\int_{0}^{t} a_{i}(u) d u}\left[1-e^{-\int_{0}^{\omega} a_{i}(u) d u}\right] & \int_{t}^{t+\omega}\left\{e^{-\int_{0}^{\xi} a_{i}(u) d u} y_{i}(\xi)\right. \\
\times & {\left[B_{i}(\xi) y_{i}(\xi)+\sum_{j=1}^{n} C_{i j}(\xi) y_{i}\left(\xi-\tau_{i j}(\xi)\right)\right.} \\
& +\sum_{j=1}^{n} D_{i j}(\xi) y_{j}\left(\xi-\gamma_{i j}(\xi)\right) \\
& \left.\left.+\sum_{j=1}^{n} E_{i j}(\xi) \int_{-\sigma_{i j}}^{0} f_{i j}(s) y_{j}(\xi+s) d s\right]\right\} d \xi
\end{aligned}
$$

which can be transformed into

$$
\begin{aligned}
y_{i}(t)=\int_{t}^{t+\omega}\left\{\frac{e^{-\int_{0}^{\xi} a_{i}(u) d u}}{e^{-\int_{0}^{t} a_{i}(u) d u}\left[1-e^{-\int_{0}^{\omega} a_{i}(u) d u}\right]} y_{i}(\xi)\right. \\
\quad \times\left[B_{i}(\xi) y_{i}(\xi)+\sum_{j=1}^{n} C_{i j}(\xi) y_{i}\left(\xi-\tau_{i j}(\xi)\right)\right. \\
+\sum_{j=1}^{n} D_{i j}(\xi) y_{j}\left(\xi-\gamma_{i j}(\xi)\right) \\
\left.\left.+\sum_{j=1}^{n} E_{i j}(\xi) \int_{-\sigma_{i j}}^{0} f_{i j}(s) y_{j}(\xi+s) d s\right]\right\} d \xi
\end{aligned}
$$

$$
\begin{aligned}
& =\int_{t}^{t+\omega}\left\{\begin{array}{l}
G_{i}(t, \xi) y_{i}(\xi) \\
\times \\
\quad\left[B_{i}(\xi) y_{i}(\xi)+\sum_{j=1}^{n} C_{i j}(\xi) y_{i}\left(\xi-\tau_{i j}(\xi)\right)\right. \\
\quad+\sum_{j=1}^{n} D_{i j}(\xi) y_{j}\left(\xi-\gamma_{i j}(\xi)\right) \\
\left.\left.\quad+\sum_{j=1}^{n} E_{i j}(\xi) \int_{-\sigma_{i j}}^{0} f_{i j}(s) y_{j}(\xi+s) d s\right]\right\} d \xi
\end{array}\right. \\
& =\int_{t}^{t+\omega} G_{i}(t, \xi)(\varphi y)_{i}(\xi) d \xi, \quad i=1,2, \ldots, n .
\end{aligned}
$$

Thus, $y_{i}(t)(i=1,2, \ldots, n)$ is a periodic solution for (15).

If $y=\left(y_{1}(t), y_{2}(t), \ldots, y_{n}(t)\right)^{T} \in X$ and $\psi y=$ $\left((\psi y)_{1},(\psi y)_{2}, \ldots,(\psi y)_{n}\right)^{T}=y$ with $y \neq 0$, then for any $t=t_{k}$ derivative the two sides of (15) about $t$,

$$
\begin{aligned}
& \frac{d\left[(\psi y)_{i}(t)\right]}{d t}=G_{i}(t, t+\omega)(\varphi y)_{i}(t+\omega)-G_{i}(t, t)(\varphi y)_{i}(t) \\
& +\int_{t}^{t+\omega} \frac{d G_{i}(t, \xi)}{d t}(\varphi y)_{i}(\xi) d \xi \\
& =-(\varphi y)_{i}(t)+a_{i}(t) \int_{t}^{t+\omega} G_{i}(t, \xi)(\varphi y)_{i}(\xi) d \xi \\
& =a_{i}(t) y_{i}(t)-(\varphi y)_{i}(t) \\
& =y_{i}(t)\left[a_{i}(t)-B_{i}(t) y_{i}(t)\right. \\
& -\sum_{j=1}^{n} C_{i j}(t) y_{i}\left(\xi-\tau_{i j}(t)\right) \\
& -\sum_{j=1}^{n} D_{i j}(t) y_{j}\left(t-\gamma_{i j}(t)\right) \\
& \left.-\sum_{j=1}^{n} E_{i j}(t) \int_{-\sigma_{i j}}^{0} f_{i j}(s) y_{j}(t+s) d s\right] \\
& =\frac{d y_{i}(t)}{d t} \text {. }
\end{aligned}
$$

Hence, $y(t)=\left(y_{1}(t), y_{2}(t), \ldots, y_{n}(t)\right)^{T} \in X$ is a positive $\omega$-periodic solution of (7). Thus we complete the proof of Lemma 8.

Our main result of this paper is as follows.

Theorem 9. Assume $\left(H_{1}\right)-\left(H_{4}\right)$. Then condition

$$
m_{0}=\min _{1 \leq i \leq n}\left\{\bar{B}_{i}+\sum_{j=1}^{n}\left(\bar{C}_{i j}+\bar{D}_{i j}+\bar{E}_{i j}\right)\right\}>0
$$


is necessary and sufficient for system (1) to have at least one positive $\omega$-periodic solution.

Proof. (Sufficiency) Let

$$
M_{0}=\max _{i \in[1, n]}\left\{\bar{B}_{i}+\sum_{j=1}^{n}\left(\bar{C}_{i j}+\bar{D}_{i j}+\bar{E}_{i j}\right)\right\},
$$

by condition (33), we know that $M_{0} \geq m_{0}>0$. Choose a constant $M \geq M_{0}$ such that $1 / \omega \beta_{i} M<1$. Let $r_{1}=1 / \omega \beta_{i} M$ and

$$
\begin{aligned}
\Omega_{r_{1}}=\{y(t) & =\left(y_{1}(t), y_{2}(t), \ldots, y_{n}(t)\right)^{T} \in X:\left|y_{i}\right|_{0} \\
& \left.<r_{1}, i=1,2, \ldots, n\right\} .
\end{aligned}
$$

For any $y=y(t) \in E \bigcap \partial \Omega_{r_{1}}, \sigma\left|y_{i}\right|_{0} \leq y_{i}(t) \leq\left|y_{i}\right|_{0}$, from (15), we obtain

$$
\begin{aligned}
& \left|(\psi y)_{i}\right|_{0} \\
& \leq \beta_{i} \int_{0}^{\omega}(\varphi y)_{i}(\xi) d \xi \\
& =\beta_{i} \int_{0}^{\omega} y_{i}(t)\left[B_{i}(t) y_{i}(t)\right. \\
& +\sum_{j=1}^{n} C_{i j}(t) y_{i}\left(\xi-\tau_{i j}(t)\right) \\
& +\sum_{j=1}^{n} D_{i j}(t) y_{j}\left(t-\gamma_{i j}(t)\right) \\
& \left.+\sum_{j=1}^{n} E_{i j}(t) \int_{-\sigma_{i j}}^{0} f_{i j}(s) y_{j}(t+s) d s\right] d \xi \\
& \leq \omega \beta_{i}\left|y_{i}\right|_{0}\left[\bar{B}_{i}+\sum_{j=1}^{n}\left(\bar{C}_{i j}+\bar{D}_{i j}+\bar{E}_{i j}\right)\right] r_{1} \\
& \leq \omega \beta_{i}\left|y_{i}\right|_{0} M_{0} r_{1} \\
& \leq\left|y_{i}\right|_{0} \text {. }
\end{aligned}
$$

Hence, for any $y=y(t) \in E \bigcap \partial \Omega_{r_{1}}, \sigma\left|y_{i}\right|_{0} \leq y_{i}(t) \leq\left|y_{i}\right|_{0}$, we have

$$
\|\psi y\|=\sum_{j=1}^{n}\left|(\psi y)_{i}\right|_{0} \leq \sum_{j=1}^{n}\left|y_{i}\right|_{0}=\|y\|,
$$

which implies that condition (1) in Lemma 4 is satisfied.

On the other hand, we choose $0<m \leq m_{0}$ such that $1 / \omega \sigma \alpha_{i} m>1$. Let $r_{2}=1 / \omega \sigma \alpha_{i} m>1$ and suppose that $u=\left(u_{1}, u_{2}, \ldots, u_{n}\right)^{T} \in E /\{0\}$. We show that for any $y=$ $y(t) \in E \bigcap \partial \Omega_{r_{2}}$ and $\lambda>0, y \neq \psi y+\lambda u$. Otherwise, there exist $y_{0}=y_{0}(t) \in E \bigcap \partial \Omega_{r_{2}}$ and $\lambda_{0}>0$, such that $y_{0}=\psi y_{0}+\lambda_{0} u$. Let $u_{i_{0}} \neq 0\left(1 \leq i_{0} \leq n\right)$, since $y_{i_{0}}(t) \geq \sigma\left|y_{i_{0}}\right|_{0}$, it follows that

$$
\begin{aligned}
& y_{i_{0}}=(\psi y)_{i_{0}}(t)+\lambda_{0} u_{i_{0}} \\
& =\int_{t}^{t+\omega} G_{i_{0}}(t, \xi)(\varphi y)_{i_{0}}(\xi) d \xi+\lambda_{0} u_{i_{0}} \\
& \geq \omega \sigma \alpha_{i}\left|y_{i_{0}}\right|_{0} \int_{0}^{\omega}\left[B_{i}(t) y_{i}(t)\right. \\
& +\sum_{j=1}^{n} C_{i j}(t) y_{i}\left(\xi-\tau_{i j}(t)\right) \\
& +\sum_{j=1}^{n} D_{i j}(t) y_{j}\left(t-\gamma_{i j}(t)\right) \\
& +\sum_{j=1}^{n} E_{i j}(t) \int_{-\sigma_{i j}}^{0} f_{i j}(s) y_{j} \\
& \times(t+s) d s] d t+\lambda_{0} u_{i_{0}} \\
& \geq \omega \sigma \alpha_{i}\left|y_{i_{0}}\right|_{0}\left[\bar{B}_{i}+\sum_{j=1}^{n}\left(\bar{C}_{i j}+\bar{D}_{i j}+\bar{E}_{i j}\right)\right] r_{2}+\lambda_{0} u_{i_{0}} \\
& \geq \omega \sigma \alpha_{i}\left|y_{i_{0}}\right|_{0} m_{0} r_{2}+\lambda_{0} u_{i_{0}} \\
& \geq\left|y_{i_{0}}\right|_{0}+\lambda_{0} u_{i_{0}}>\left|y_{i_{0}}\right|_{0},
\end{aligned}
$$

which is a contradiction. This proves that condition (2) in Lemma 4 is also satisfied. By Lemmas 4 and 8 , system (7) has at least one positive $\omega$-periodic solution. From Lemma 2, system (1) has at least one positive $\omega$-periodic solution.

(Necessity) Suppose that (33) does not hold. Then there exists at least an $i_{0}\left(1 \leq i_{0} \leq n\right)$ such that

$$
\bar{B}_{i_{0}}=0, \quad \bar{C}_{i_{0} j}=\bar{D}_{i_{0} j}=\bar{E}_{i_{0} j}=0, \quad j \in[1, n] .
$$

If system (7) has a positive $\omega$-periodic solution $y(t)=\left(y_{1}(t)\right.$, $\left.y_{2}(t), \ldots, y_{n}(t)\right)^{T}$, then we have

$$
\frac{d y_{i_{0}}(t)}{d t}=a_{i_{0}}(t) y_{i_{0}}(t)
$$

Integrating the previos equation over $[t, t+\omega]$, we can have

$$
0=\ln \frac{y_{i_{0}}(t+\omega)}{y_{i_{0}}(t)}=\int_{0}^{\omega} a_{i_{0}}(t) d t>0,
$$

which is a contradiction. The proof of Theorem 9 is complete.

\section{Existence of Periodic Solution of (2)}

Now, we are at the position to study the existence of positive periodic solutions of (2). By carrying out similar arguments 
as in Section 2, it is not difficult to derive sufficient criteria for the existence of positive periodic solutions of (2). For simplicity, we prefer to list later the corresponding criteria for (2) without proof since the proofs are very similar to those in Section 2 .

For $(t, \xi) \in R^{2}, 1 \leq i \leq n$, we define

$$
\begin{array}{r}
G_{i}(t, \xi)=\frac{e^{-\int_{t}^{\xi} a_{i}(u)} d u}{1-e^{-\int_{0}^{\omega} a_{i}(u)} d u}=\frac{e^{\int_{\xi}^{t+\omega} a_{i}(u)} d u}{e^{\int_{0}^{\omega} a_{i}(u)} d u-1}, \\
G(t, \xi)=\operatorname{diag}\left[G_{1}(t, \xi), G_{2}(t, \xi), \ldots, G_{n}(t, \xi)\right], \\
t \leq \xi \leq t+\omega .
\end{array}
$$

It is clear that $G_{i}(t+\omega, \xi+\omega)=G_{i}(t, s), \partial G_{i}(t, \xi) / \partial t=$ $a_{i}(t) G_{i}(t, \xi), G_{i}(t, t)-G_{i}(t, t+\omega)=1$. In view of $\left(\mathrm{H}_{1}\right)$, we also define for $1 \leq i \leq n$

$$
\begin{gathered}
\alpha_{i}:=\min _{0 \leq t \leq \xi \leq \omega}\left|G_{i}(t, \xi)\right|=\frac{1}{e^{\int_{0}^{\omega} a_{i}(u) d u}-1}, \\
\beta_{i}:=\max _{0 \leq t \leq \xi \leq \omega}\left|G_{i}(t, \xi)\right|=\frac{e^{\int_{0}^{\omega} a_{i}(u) d u}}{e^{\int_{0}^{\omega} a_{i}(u) d u}-1}, \\
\alpha=\min _{1 \leq i \leq n} \alpha_{i}, \quad \beta=\max _{1 \leq i \leq n} \beta_{i}, \quad \sigma=\frac{\alpha}{\beta} \in(0,1) .
\end{gathered}
$$

Let $X=\left\{y=\left(y_{1}(t), y_{2}(t), \ldots, y_{n}(t)\right)^{T} \in P C\left(R, R^{n}\right)\right.$ | $y(t+\omega)=y(t)\}$ with the norm $\|y\|=\sum_{i=1}^{n}\left|y_{i}\right|_{0},\left|y_{i}\right|_{0}=$ $\sup _{t \in[0, \omega]}\left|y_{i}(t)\right|$. It is easy to verify that $(X,\|\cdot\|)$ is a Banach space. Define $P$ to be a cone in $X$ by

$$
\begin{aligned}
P=\{y & =\left(y_{1}(t), y_{2}(t), \ldots, y_{n}(t)\right)^{T} \in X: y_{i}(t) \\
& \left.\geq \delta\left\|y_{i}\right\|_{0}, t \in[0, \omega]\right\} .
\end{aligned}
$$

We easily verify that $P$ is a cone in $X$. We define an operator $A: P \rightarrow X$ as follows:

$$
(A y)(t)=\left((A y)_{1}(t),(A y)_{2}(t), \ldots,(A y)_{n}(t)\right)^{T}
$$

where

$$
(A y)_{i}(t)=\int_{t}^{t+\omega} G_{i}(t, \xi)(\phi y)_{i}(\xi) d s,
$$

where

$$
\begin{aligned}
&(\emptyset y)_{i}(t)=y_{i}(t)[ B_{i}(t) y_{i}(t)-\sum_{j=1}^{n} C_{i j}(t) y_{i}\left(\xi-\tau_{i j}(t)\right) \\
&+\sum_{j=1}^{n} D_{i j}(t) y_{j}\left(t-\gamma_{i j}(t)\right) \\
&\left.+\sum_{j=1}^{n} E_{i j}(t) \int_{-\sigma_{i j}}^{0} f_{i j}(s) y_{j}(t+s) d s\right], \\
& i=1,2, \ldots, n .
\end{aligned}
$$

The proof of the following lemmas and theorems is similar to those in the Section 2, so we all omit the details here.
Lemma 10. Assume that $\left(H_{1}\right)-\left(H_{4}\right)$ hold. Then the solutions of (2) are defined on $[-\tau, \infty)$ and are positive.

Lemma 11. Assume that $\left(H_{1}\right)-\left(H_{4}\right)$ hold. Then $A: E \rightarrow E$ is well defined.

Lemma 12. The operator $A: E \rightarrow E$ is continuous and completely continuous.

Lemma 13. Assume that $\left(H_{1}\right)-\left(H_{4}\right)$ hold. The existence of positive $\omega$-periodic solution of (8) is equivalent to that of nonzero fixed point of $A$ in $P$.

Theorem 14. Assume $\left(H_{1}\right)-\left(H_{4}\right)$. Moreover, if the condition

$$
l_{0}=\min _{1 \leq i \leq n}\left\{\bar{B}_{i}+\sum_{j=1}^{n}\left(-\bar{C}_{i j}+\bar{D}_{i j}+\bar{E}_{i j}\right)\right\}>0
$$

holds, then the system (2) has at least one positive w-periodic solution.

\section{Applications}

In this section, as some applications of our main results, we will consider some special cases of systems (1) and (2), which have been investigated extensively in the literature.

Application 1. We consider the following periodic singlespecies population growth models with periodic delay and impulse:

$$
\begin{gathered}
y^{\prime}(t)=y(t)[r(t)-a(t) y(t)-b(t) y(t-\tau(t))], \\
t \in R, t \neq t_{k}, \\
\Delta y\left(t_{k}\right)=\theta_{k} y\left(t_{k}\right), \quad k \in Z_{+},
\end{gathered}
$$

which is a special case of system (1), and where $a(t), b(t)$, $\tau(t) \in C\left(R, R_{+}\right)$are $\omega$-periodic. Thus from Theorem 9 we have the following.

Theorem 15. Assume that $\left(H_{1}\right)-\left(H_{4}\right)$ hold. Then condition:

$$
R_{1}=\bar{A}+\bar{B}>0
$$

is necessary and sufficient for system (49) to have at least one positive $\omega$-periodic solution, where

$$
\begin{gathered}
A(t)=a(t) \prod_{0<t_{k}<t}\left(1+\theta_{k}\right), \\
B(t)=b(t) \prod_{0<t_{k}<t-\tau(t)}\left(1+\theta_{k}\right) .
\end{gathered}
$$

Application 2. We consider the following periodic singlespecies population growth models with periodic delay and impulse:

$$
\begin{gathered}
y^{\prime}(t)=y(t)[r(t)-a(t) y(t)+b(t) y(t-\tau(t))], \\
t \in R, t \neq t_{k}, \\
\Delta y\left(t_{k}\right)=\theta_{k} y\left(t_{k}\right), \quad k \in Z_{+},
\end{gathered}
$$


which is a special case of system (2), and where $a(t), b(t)$, $\tau(t) \in C\left(R, R_{+}\right)$are $\omega$-periodic. Thus from Theorem 14 we have the following.

Theorem 16. Assume that $\left(H_{1}\right)-\left(H_{4}\right)$ hold. Moreover, if the condition

$$
R_{2}=\bar{A}-\bar{B}>0
$$

holds, the system (52) has at least one positive $\omega$-periodic solution, where

$$
\begin{gathered}
A(t)=a(t) \prod_{0<t_{k}<t}\left(1+\theta_{k}\right), \\
B(t)=b(t) \prod_{0<t_{k}<t-\tau(t)}\left(1+\theta_{k}\right) .
\end{gathered}
$$

Application 3. We study the following two-species competitive system with impulses:

$$
\begin{array}{r}
y_{1}^{\prime}(t)=y_{1}(t)\left[r_{1}(t)-a_{1}(t) y_{1}(t)-c_{1}(t) y_{2}(t)\right], \\
y_{2}^{\prime}(t)=y_{2}(t)\left[r_{2}(t)-a_{2}(t) y_{2}(t)-c_{2}(t) y_{1}(t)\right], \\
t \neq t_{k}, \\
\Delta y_{i}\left(t_{k}\right)=\theta_{k} y\left(t_{k}\right), \quad i=1,2, k \in Z_{+}, t=t_{k},
\end{array}
$$

which is a special case of system (1), and where $a_{i}(t), c_{i}(t) \epsilon$ $C\left(R, R_{+}\right)$are $\omega$-periodic. Thus from Theorem 9 we have the following.

Theorem 17. Assume that $\left(H_{1}\right)-\left(H_{4}\right)$ hold. Then condition

$$
R_{3}=\min _{1 \leq i \leq 2}\left\{\bar{A}_{i}+\bar{C}_{i}\right\}>0
$$

is necessary and sufficient for system (55) to have at least one positive $\omega$-periodic solution, where

$$
\begin{array}{r}
A_{i}(t)=a_{i}(t) \prod_{0<t_{k}<t}\left(1+\theta_{k}\right), \\
C_{i}(t)=c_{i}(t) \prod_{0<t_{k}<t}\left(1+\theta_{k}\right), \\
i=1,2 .
\end{array}
$$

Application 4. We study the following two-species competitive system with impulses:

$$
\begin{aligned}
y_{1}^{\prime}(t)=y_{1}(t)[ & r_{1}(t)-a_{1}(t) y_{1}(t) \\
& -\sum_{j=1}^{n} b_{1 j}(t) y_{1}\left(t-\tau_{j}(t)\right) \\
& \left.-\sum_{j=1}^{n} c_{1 j}(t) y_{2}\left(t-\rho_{j}(t)\right)\right],
\end{aligned}
$$

$$
\begin{array}{r}
y_{2}^{\prime}(t)=y_{2}(t)\left[r_{2}(t)-a_{2}(t) y_{2}(t)\right. \\
-\sum_{j=1}^{n} b_{2 j}(t) y_{2}\left(t-\eta_{j}(t)\right) \\
\left.-\sum_{j=1}^{n} c_{2 j}(t) y_{1}\left(t-\sigma_{j}(t)\right)\right], \\
\Delta y_{i}\left(t_{k}\right)=\theta_{k} y\left(t_{k}\right), \quad i=1,2, k \in Z_{+}, \quad t=t_{k},
\end{array}
$$

which is a special case of system (1), and where $a_{i}(t)$, $b_{i j}(t), c_{i j}(t) \in C\left(R, R_{+}\right), i=1,2$, are $\omega$-periodic. Thus from Theorem 9 we have the following.

Theorem 18. Assume that $\left(H_{1}\right)-\left(H_{4}\right)$ hold. Then condition

$$
R_{4}=\min _{1 \leq i \leq 2}\left\{\bar{A}_{i}+\sum_{i=1}^{n}\left(\bar{B}_{i j}+\bar{C}_{i j}\right)\right\}>0
$$

is necessary and sufficient for system (58) to have at least one positive $\omega$-periodic solution, where

$$
\begin{gathered}
A_{i}(t)=a_{i}(t) \prod_{0<t_{k}<t}\left(1+\theta_{k}\right), \\
B_{1 j}(t)=b_{1 j}(t) \prod_{0<t_{k}<t-\tau_{j}(t)}\left(1+\theta_{k}\right), \\
B_{2 j}(t)=b_{2 j}(t) \prod_{0<t_{k}<t-\eta_{j}(t)}\left(1+\theta_{k}\right), \\
C_{1 j}(t)=C_{1 j}(t) \prod_{0<t_{k}<t-\rho_{j}(t)}\left(1+\theta_{k}\right), \\
C_{2 j}(t)=c_{2 j}(t) \prod_{0<t_{k}<t-\sigma_{j}(t)}\left(1+\theta_{k}\right), \\
i=1,2 .
\end{gathered}
$$

Application 5. We study the following two-species competitive system with impulses:

$$
\begin{aligned}
y_{1}^{\prime}(t)=y_{1}(t)[ & r_{1}(t)-a_{1}(t) y_{1}(t) \\
& +\sum_{j=1}^{n} b_{1 j}(t) y_{1}\left(t-\tau_{j}(t)\right) \\
& \left.-\sum_{j=1}^{n} c_{1 j}(t) y_{2}\left(t-\rho_{j}(t)\right)\right],
\end{aligned}
$$




$$
\begin{aligned}
& y_{2}^{\prime}(t)=y_{2}(t)\left[r_{2}(t)-a_{2}(t) y_{2}(t)\right. \\
& +\sum_{j=1}^{n} b_{2 j}(t) y_{2}\left(t-\eta_{j}(t)\right) \\
& \left.-\sum_{j=1}^{n} c_{2 j}(t) y_{1}\left(t-\sigma_{j}(t)\right)\right] \text {, } \\
& t \neq t_{k} \text {, } \\
& \Delta y_{i}\left(t_{k}\right)=\theta_{k} y\left(t_{k}\right), \quad i=1,2, k \in Z_{+}, t=t_{k} \text {, }
\end{aligned}
$$

which is a special case of system (2), and where $a_{i}(t)$, $b_{i j}(t), c_{i j}(t) \in C\left(R, R_{+}\right), i=1,2$, are $\omega$-periodic. Thus from Theorem 14 we have the following.

Theorem 19. Assume that $\left(H_{1}\right)-\left(H_{4}\right)$ hold. Moreover, if the condition

$$
R_{5}=\min _{1 \leq i \leq 2}\left\{\bar{A}_{i}+\sum_{i=1}^{n}\left(-\bar{B}_{i j}+\bar{C}_{i j}\right)\right\}>0
$$

holds, the system (61) has at least one positive $\omega$-periodic solution, where

$$
\begin{gathered}
A_{i}(t)=a_{i}(t) \prod_{0<t_{k}<t}\left(1+\theta_{k}\right), \\
B_{1 j}(t)=b_{1 j}(t) \prod_{0<t_{k}<t-\tau_{j}(t)}\left(1+\theta_{k}\right), \\
B_{2 j}(t)=b_{2 j}(t) \prod_{0<t_{k}<t-\eta_{j}(t)}\left(1+\theta_{k}\right), \\
C_{1 j}(t)=C_{1 j}(t) \prod_{0<t_{k}<t-\rho_{j}(t)}\left(1+\theta_{k}\right), \\
C_{2 j}(t)=c_{2 j}(t) \prod_{0<t_{k}<t-\sigma_{j}(t)}\left(1+\theta_{k}\right), \\
i=1,2 .
\end{gathered}
$$

Application 6. We investigate the following n-species competitive systems with impulses:

$$
\begin{aligned}
x_{i}^{\prime}(t)=x_{i}(t)[ & a_{i}(t)-b_{i}(t) x_{i}(t) \\
& -\sum_{j=1}^{n} c_{i j}(t) x_{j}\left(t-\tau_{i j}(t)\right)-\sum_{j=1}^{n} d_{i j}(t) \\
& \left.\times \int_{-\sigma_{i j}}^{0} K_{i j}(s) x_{j}(t+s) d s\right], \quad t \neq t_{k}, \\
x_{i}\left(t_{k}^{+}\right)-x_{i}\left(t_{k}^{-}\right) & =\theta_{i k} x_{i}\left(t_{k}\right), \quad i=1,2, \ldots, n, k \in Z_{+},
\end{aligned}
$$

which is a special case of system (1), and where $a_{i}(t), b_{i}(t)$, $c_{i j}(t), d_{i j}(t), \tau_{i j}(t) \in C\left(R, R_{+}\right), i=1,2, \ldots, n$, are $\omega$-periodic. Moreover, $K_{i j} \in P C, \sigma_{i j}>0$ is a constant and $\int_{-\sigma_{i j}}^{0} K_{i j}(s) d s=$ 1. Thus from Theorem 9 we have the following.

Theorem 20. Assume that $\left(H_{1}\right)-\left(H_{4}\right)$ hold. Then condition

$$
R_{6}=\min _{1 \leq i \leq 2}\left\{\bar{B}_{i}+\sum_{i=1}^{n}\left(\bar{C}_{i j}+\bar{D}_{i j}\right)\right\}>0
$$

is necessary and sufficient for system (64) to have at least one positive $\omega$-periodic solution, where

$$
\begin{gathered}
B_{i}(t)=b_{i}(t) \prod_{0<t_{k}<t}\left(1+\theta_{i k}\right), \\
C_{i j}(t)=C_{i j}(t) \prod_{0<t_{k}<t-\tau_{i j}(t)}\left(1+\theta_{i k}\right), \\
D_{i j}(t)=d_{i j}(t) \prod_{0<t_{k}<t-\sigma_{i j}}\left(1+\theta_{i k}\right) .
\end{gathered}
$$

Remark 21. We apply the main results obtained in the previous section to study some examples which have some biological implications. A very basic and important ecological problem associated with the study of population is that of the existence of positive periodic solutions which play the role played by the equilibrium of the autonomous models, meaning that the species is in an equilibrium state. From Theorems 15-20, we see that under the appropriate conditions, the impulsive perturbations do not affect the existence of periodic solution of systems.

\section{Acknowledgments}

This work was supported by the Construct Program of the Key Discipline in Hunan province, NSF of China (nos. 10971229, 11161015), PSF of China (2012M512162), and NSF of Hunan province (nos. 11JJ9002, 12JJ9001, and 13JJ4098).

\section{References}

[1] D. D. Bainov and P. S. Simeonov, System With Impulsive Effect: Stability, Theory and Applications, John Wiley \& Sons, New York, NY, USA, 1989.

[2] V. Lakshmikantham, D. D. Baĭnov, and P. S. Simeonov, Theory of Impulsive Differential Equations, vol. 6 of Series in Modern Applied Mathematics, World Scientific, Teaneck, NJ, USA, 1989.

[3] A. M. Samoikleno and N. A. Perestyuk, Impulsive Differential Equations, vol. 28, World Scientific, Singapore, 1995.

[4] D. Bainov and P. Simeonov, Oscillation Theory of Impulsive Differential Equations, International Publications, 1998.

[5] J. R. Yan and A. M. Zhao, "Oscillation and stability of linear impulsive delay differential equations," Journal of Mathematical Analysis and Applications, vol. 227, no. 1, pp. 187-194, 1998.

[6] G. Ballinger and X. Liu, "Existence, uniqueness and boundedness results for impulsive delay differential equations," Applicable Analysis, vol. 74, no. 1-2, pp. 71-93, 2000. 
[7] M. S. Peng and W. G. Ge, "Oscillation criteria for second-order nonlinear differential equations with impulses," Computers \& Mathematics with Applications, vol. 39, no. 5-6, pp. 217-225, 2000.

[8] B. Liu and J. Yu, "Existence of solution of $m$-point boundary value problems of second-order differential systems with impulses," Applied Mathematics and Computation, vol. 125, no. 2-3, pp. 155-175, 2002.

[9] X. N. Liu and L. S. Chen, "Complex dynamics of Holling type II Lotka-Volterra predator-prey system with impulsive perturbations on the predator," Chaos, Solitons and Fractals, vol. 16, no. 2, pp. 311-320, 2003.

[10] H.-F. Huo, W.-T. Li, and X. Liu, "Existence and global attractivity of positive periodic solution of an impulsive delay differential equation," Applicable Analysis, vol. 83, no. 12, pp. 1279-1290, 2004.

[11] J. Yan, "Existence of positive periodic solutions of impulsive functional differential equations with two parameters," Journal of Mathematical Analysis and Applications, vol. 327, no. 2, pp. 854-868, 2007.

[12] M. Z. He, Z. Li, and F. D. Chen, "Permanence, extinction and global attractivity of the periodic Gilpin-Ayala competition system with impulses," Nonlinear Analysis. Real World Applications. An International Multidisciplinary Journal, vol. 11, no. 3, pp. 1537-1551, 2010.

[13] M. E. Gilpin and F. J. Ayala, "Global model of growth and competition," Proceedings of the National Academy of Sciences United States of America, vol. 70, no. 2, pp. 3590-3593, 1973.

[14] M. Fan and K. Wang, "Periodic solutions of single population model with hereditary effect," Mathematica Applicata, vol. 13, no. 2 , pp. 58-61, 2000.

[15] H. I. Freedman and J. H. Wu, "Periodic solutions of singlespecies models with periodic delay," SIAM Journal on Mathematical Analysis, vol. 23, no. 3, pp. 689-701, 1992.

[16] C. Alvarez and A. C. Lazer, "An application of topological degree to the periodic competing species problem," Australian Mathematical Society, vol. 28, no. 2, pp. 202-219, 1986.

[17] S. Ahmad, "Convergence and ultimate bounds of solutions of the nonautonomous Volterra-Lotka competition equations," Journal of Mathematical Analysis and Applications, vol. 127, no. 2, pp. 377-387, 1987.

[18] Z. Liu, M. Fan, and L. Chen, "Globally asymptotic stability in two periodic delayed competitive systems," Applied Mathematics and Computation, vol. 197, no. 1, pp. 271-287, 2008.

[19] D. J. Guo, Nonlinear Functional Analysis, ShanDong Science and Technology Press, 2001.

[20] M. A. Krasnoselskii, Positive Solution of Operator Equation, Noordhoff, Grőningen, The Netherlands, 1964.

[21] K. Deimling, Nonlinear Functional Analysis, Springer, Berlin, Germany, 1985.

[22] D. J. Guo and V. Lakshmikantham, Nonlinear Problems in Abstract Cones, vol. 5 of Notes and Reports in Mathematics in Science and Engineering, Academic Press, Boston, Mass, USA, 1988. 


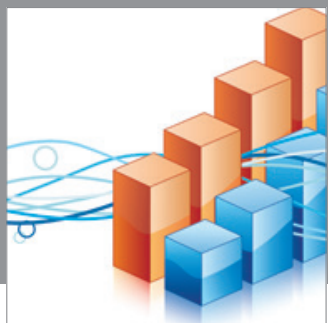

Advances in

Operations Research

mansans

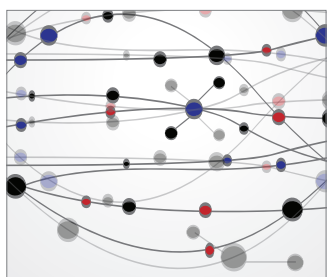

The Scientific World Journal
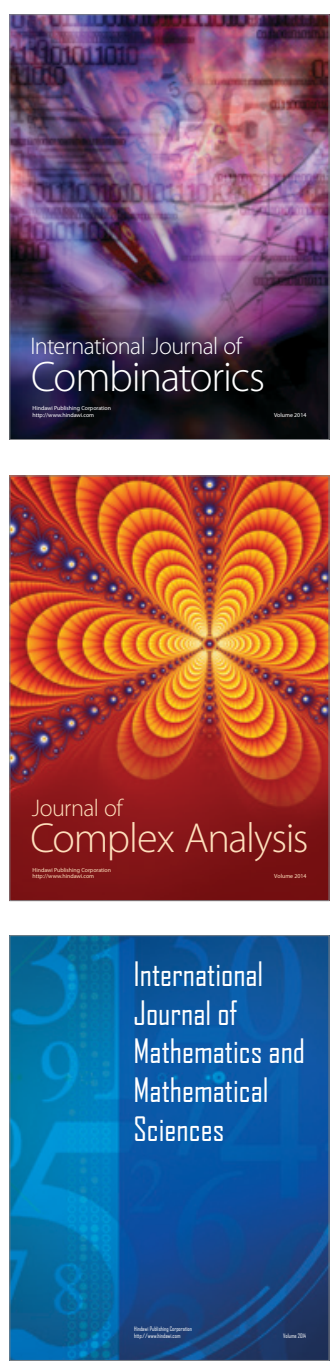
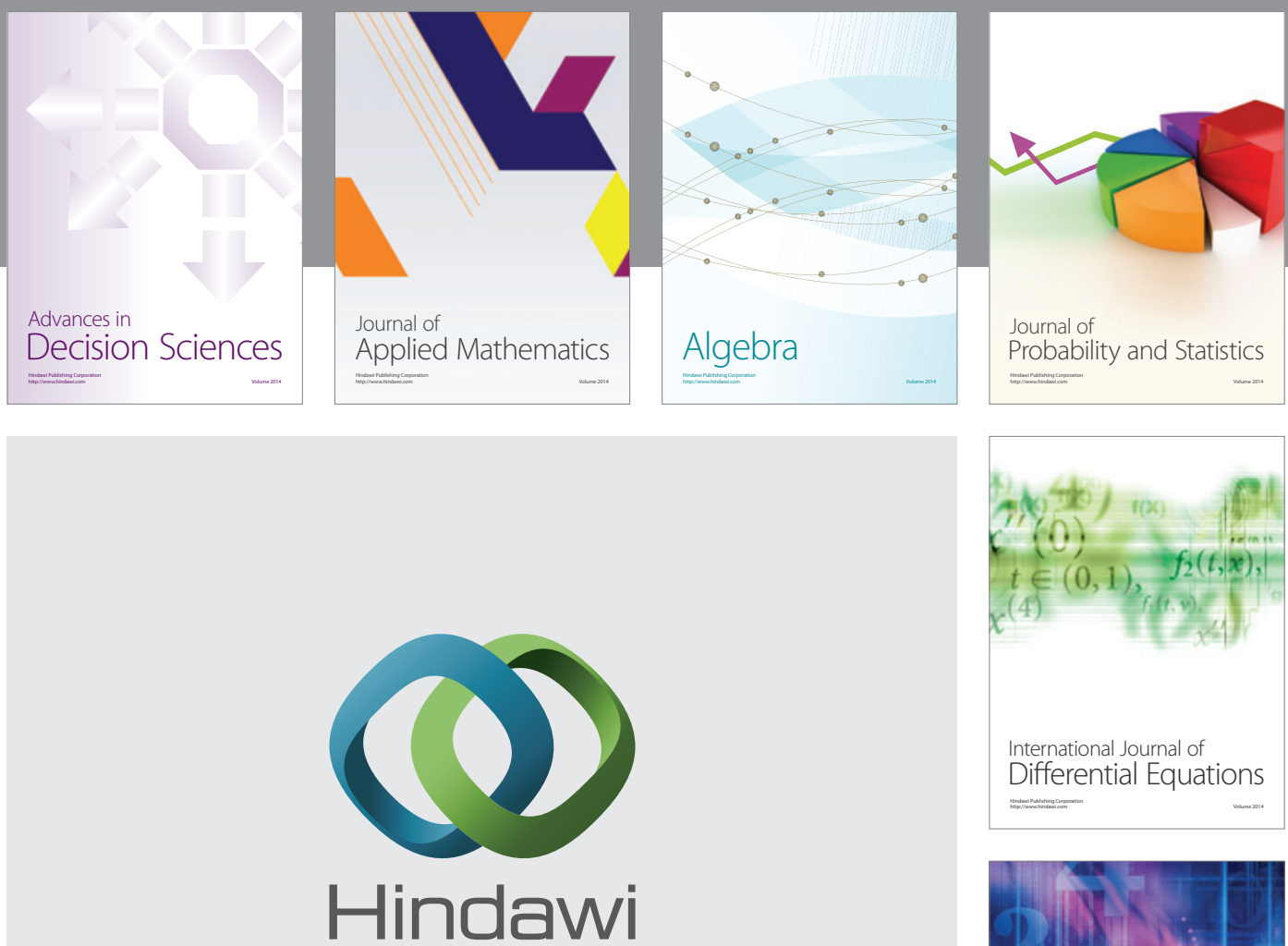

Submit your manuscripts at http://www.hindawi.com
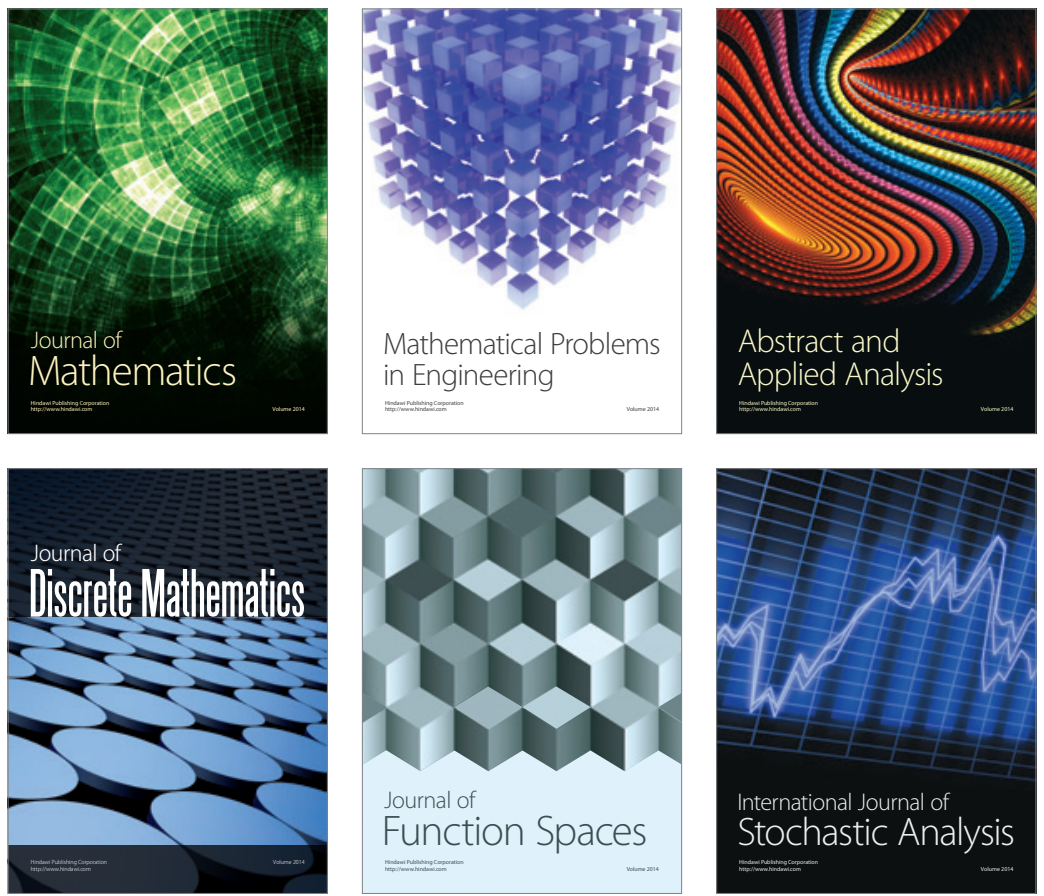

Journal of

Function Spaces

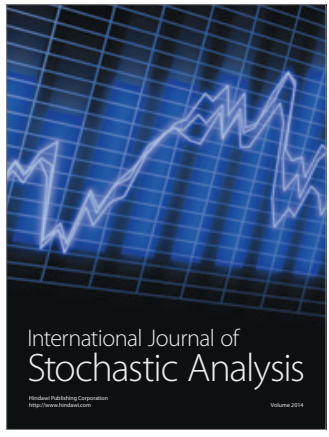

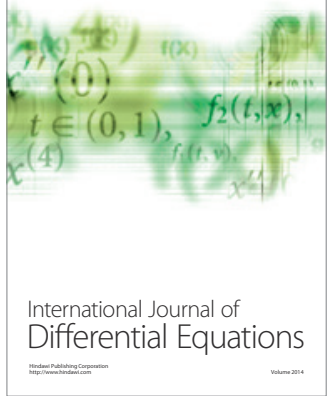
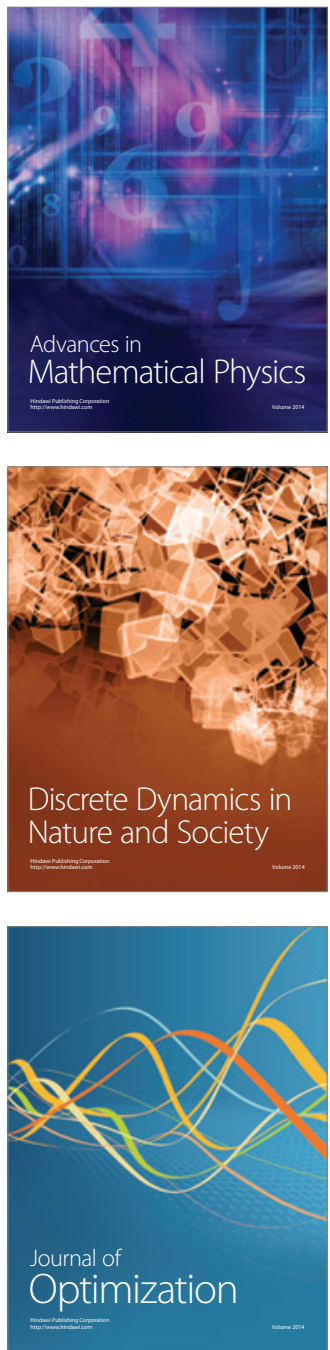